\title{
Everolimus-eluting bioresorbable scaffolds in patients with coronary artery disease: results from the German-Austrian ABSORB ReglstRy (GABI-R)
}

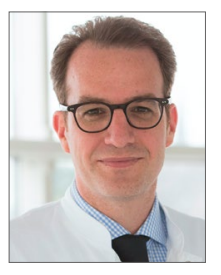

Holger M. Nef ${ }^{1 *}$, MD; Jens Wiebe ${ }^{2}$, MD; Johannes Kastner ${ }^{3}$, MD; Julinda Mehilli' ${ }^{4}$, MD; Thomas Münzel ${ }^{5}$, MD; Christoph Naber ${ }^{6}$, MD; Till Neumann ${ }^{7}, \mathrm{MD}, \mathrm{PhD}$; Gert Richardt ${ }^{8}$, MD; Axel Schmermund 9 , MD; Jochen Wöhrle ${ }^{10}$, MD; Ralf Zahn ${ }^{11}$, MD; Thomas Riemer ${ }^{12}$, PhD; Stephan Achenbach ${ }^{13}$, MD; Christian W. Hamm ${ }^{1,14}$, MD; for the GABI-R Study Group

1. Medizinische Klinik I, Department of Cardiology, University of Giessen, Giessen, Germany; 2. Deutsches Herzzentrum München, Munich, Germany; 3. Department of Cardiology, University of Vienna Medical School, Vienna, Austria; 4. Department of Cardiology, Munich University Clinic, Ludwig Maximilian University of Munich, Munich, Germany; 5. Department of Medicine II, University Medical Center, Johannes Gutenberg University Mainz, Mainz, Germany; 6. Klinik für Kardiologie und Angiologie, Elisabeth-Krankenhaus, Essen, Germany; 7. Department of Cardiology, University of Essen, Essen, Germany; 8. Herzzentrum, Segeberger Kliniken GmbH, Bad Segeberg, Germany; 9. Cardioangiologisches Centrum Bethanien, Frankfurt am Main, Germany; 10. Department of Internal Medicine II, University of Ulm, Ulm, Germany; 11. Abteilung für Kardiologie, Herzzentrum Ludwigshafen, Ludwigshafen, Germany; 12. Institut für Herzinfarktforschung, Ludwigshafen, Germany; 13. Medizinische Klinik 2, Friedrich-Alexander Universität Erlangen-Nürnberg, Erlangen, Germany; 14. Department of Cardiology, Kerckhoff Heart and Thorax Center, Bad Nauheim, Germany

H.M. Nef and J. Wiebe contributed equally to this manuscript.

\section{KEYWORDS}

- acute coronary

syndrome/

non-ST-elevation

acute coronary

syndrome

(ACS/NSTE-ACS)

- bioresorbable

scaffolds

- non-ST-elevation

myocardial

infarction

(NSTEMI)

- stable angina

- ST-elevation

myocardial

infarction (STEMI)

- stent thrombosis

\begin{abstract}
Aims: The aim of this study was to analyse the procedural results and midterm safety of everolimus-eluting bioresorbable vascular scaffolds (BVS) used for percutaneous coronary intervention in a large all-comers cohort from the German-Austrian ABSORB RegIstRy (GABI-R).

Methods and results: A total of 3,231 patients were included in this prospective, observational, multicentre study (ClinicalTrials.gov NCT02066623) of consecutive patients undergoing BVS implantation between November 2013 and January 2016. Endpoints were major adverse cardiac events (MACE; a composite endpoint of death, target vessel revascularisation, and myocardial infarction), and target lesion failure (TLF; a composite endpoint of cardiac death, target vessel myocardial infarction, and target lesion revascularisation). Scaffold thrombosis was a further endpoint. Of all patients, $51.5 \%$ presented with acute coronary syndrome. Predilatation and post-dilatation were performed in $91.5 \%$ and $71.9 \%$ of patients, respectively. Procedural success was $98.9 \%$. After six months, the incidence of MACE was $4.1 \%$ and of TLF $2.4 \%$. The rate of target vessel MI was $1.5 \%$, and target lesion revascularisation was performed in $1.8 \%$. Definite/ probable scaffold thrombosis was documented in $1.4 \%$ of patients.
\end{abstract}

Conclusions: GABI-R, the largest registry to provide data regarding safety after BVS implantation in a real-world setting, reveals high procedural success and low six-month event rates.

*Corresponding author: Medizinische Klinik I, Department of Cardiology, University of Giessen, Klinikstrasse 33, 35392 Giessen, Germany. E-mail: holger.nef@innere.med.uni-giessen.de 


\section{Abbreviations}

$\begin{array}{ll}\text { ARC } & \text { Academic Research Consortium } \\ \text { BVS } & \text { bioresorbable vascular scaffold(s) } \\ \text { DES } & \text { drug-eluting stent } \\ \text { MACE } & \text { major adverse cardiac events } \\ \text { MI } & \text { myocardial infarction } \\ \text { TLF } & \text { target lesion failure } \\ \text { TLR } & \text { target lesion revascularisation } \\ \text { TVF } & \text { target vessel failure } \\ \text { TVR } & \text { target vessel revascularisation }\end{array}$

\section{Introduction}

Several randomised controlled trials have demonstrated that polyL-lactic acid-based everolimus-eluting bioresorbable vascular scaffolds (BVS; Abbott Vascular, Santa Clara, CA, USA) appear to be similar in terms of safety and effectiveness compared to currentgeneration metallic drug-eluting stents (DES) for the percutaneous treatment of coronary artery stenosis, despite a higher risk of scaffold thrombosis ${ }^{1}$. Two recent trials have shown significantly more events during long-term follow-up ${ }^{2,3}$. However, most randomised studies share strict inclusion criteria, and consequently a large proportion of patients are underrepresented or even excluded. Nevertheless, it is essential to verify the results of these trials in a real-world setting.

The retrospective GHOST-EU registry, which is the largest allcomers registry on BVS to date, also demonstrated unexpectedly high event rates ${ }^{4}$. However, it has been shown that lesion morphology and implantation techniques may influence clinical outcomes ${ }^{5,6}$.

The present interim analysis of the prospective German-Austrian ABSORB RegIstRy (GABI-R) provides detailed procedural results and midterm safety outcomes of the largest prospective cohort of patients treated with BVS in daily clinical practice.

\section{Editorial, see page 1259}

\section{Methods}

\section{STUDY DESIGN AND PATIENT COHORT}

GABI-R is a prospective, observational, multicentre study (ClinicalTrials.gov NCT02066623) of consecutive patients undergoing BVS implantation at 92 sites in Germany and Austria between November 2013 and January 2016. This investigation was approved by the local ethics boards. All patients received and signed a written consent. Data sets were collected centrally via an electronic case report form provided by the IHF (Institut für Herzinfarktforschung, Ludwigshafen, Germany); thus, source verification and quality control were conducted independently. Monitoring was performed once in 30 participating clinics, which included at least 10 patients each. The informed consent was checked in every case and all source data were verified in five patients. Follow-up via questionnaire or telephone interview was predefined by protocol after 30 days, six months, two and five years. Additional details have been published previously?

\section{PROCEDURAL DETAILS}

The implantation technique complied with the current standards and, albeit not mandatory, predilatation and post-dilatation were strongly recommended ${ }^{8}$. The use of intravascular imaging was left to the implanting physician's discretion. High-pressure balloon dilatation was defined as dilatation at $\geq 14 \mathrm{~atm}$. Procedural success was defined as a visually estimated residual stenosis $<30 \%$ within the treated segment. Dual antiplatelet therapy was prescribed for at least one year in all patients.

\section{TARGET PARAMETERS}

The primary endpoints were major adverse cardiac events (MACE), including cardiac death, target vessel revascularisation (TVR), or myocardial infarction (MI), and target lesion failure (TLF), including cardiac death, clinically driven target lesion revascularisation (TLR), or target vessel MI. The composite endpoint of target vessel failure (TVF) comprises cardiac death, target vessel MI, and clinically driven target vessel revascularisation. Clinically driven TLR was defined as $\geq 50 \%$ diameter stenosis and recurrent angina, objective signs of ischaemia, abnormal results of invasive functional testing or $\geq 70 \%$ diameter stenosis even in the absence of the previously noted criteria ${ }^{9}$. Scaffold thrombosis, according to the Academic Research Consortium (ARC) criteria, was also evaluated ${ }^{9}$. Cardiac death was defined as death from an immediate cardiac cause or complications related to the procedure or any death in which a cardiac cause could not be excluded. MI was defined according to the World Health Organization extended definition ${ }^{10}$.

All events were adjudicated by an independent events committee based on clinical charts and review of the angiograms. There was no quantitative core laboratory involved for adjudication. The events committee was not involved in the study.

\section{STATISTICAL ANALYSIS}

Qualitative data were analysed as absolute values and percentages, and continuous variables are presented as means with standard deviation. Time-to-event data were visualised using Kaplan-Meier (product-limit) estimates. P-values for the homogeneity of timeto-event (survival) curves were calculated by the log-rank test. A small number of missing event dates were completed by random hot-deck imputation (one imputed date used in this analysis). In addition, a comparison was made between patients treated in 2014 and patients treated in 2015 and later. Continuous data were compared with the Wilcoxon rank test, categorical data with the chi-square test and Kaplan-Meier estimates with the log-rank test.

\section{Results \\ BASELINE CHARACTERISTICS}

A total of 3,231 patients with a mean age of $60.9 \pm 11.0$ years and $23.2 \%$ being female were included. Diabetes was present in $21.0 \%$, hypertension was noted in $73.3 \%$ and hyperlipidaemia in $56.7 \%$. An acute coronary syndrome at hospital admission was present in $51.5 \%$. Further baseline characteristics are presented in Table 1.

A total of 4,383 lesions were treated. Mean visually estimated lesion length was $17.1 \pm 9.2 \mathrm{~mm}$, and $6.7 \%$ of patients had lesions $>34 \mathrm{~mm}$. Of all lesions, $36.5 \%$ were classified B2/C according to the ACC/AHA classification and bifurcated lesions were present in $2.9 \%$. Further lesion-related characteristics can be found in Table 2. 
Table 1. Baseline characteristics.

\begin{tabular}{|l|c|}
\hline Patients & 3,231 \\
\hline Female gender & $23.2(749 / 3,231)$ \\
\hline Age, years & $60.9 \pm 11.0$ \\
\hline Diabetes mellitus & $21.0(672 / 3,201)$ \\
\hline \multicolumn{1}{|c|}{ insulin-treated } & $34.9(221 / 633)$ \\
\hline Current or previous smoker & $58.1(1,780 / 3,062)$ \\
\hline Hyperlipoproteinaemia & $56.7(1,752 / 3,091)$ \\
\hline Family history of coronary artery disease & $40.6(1,153 / 2,839)$ \\
\hline Hypertension & $73.3(2,333 / 3,184)$ \\
\hline Renal failure & $8.0(257 / 3,206)$ \\
\hline Atrial fibrillation & $6.8(218 / 3,183)$ \\
\hline Previous myocardial infarction & $22.4(713 / 3,178)$ \\
\hline Prior bypass surgery & $2.4(78 / 3,216)$ \\
\hline Prior coronary intervention & $35.6(985 / 2,770)$ \\
\hline Acute coronary syndrome & $51.5(1,663 / 3,228)$ \\
\hline ST-elevation myocardial infarction & $33.7(560 / 1,663)$ \\
\hline Non-ST-elevation myocardial infarction & $43.5(724 / 1,663)$ \\
\hline Unstable angina & $22.8(379 / 1,663)$ \\
\hline Stable angina & $33.3(1,075 / 3,228)$ \\
\hline Silent myocardial ischaemia & $4.3(138 / 3,228)$ \\
\hline Other & $4.3(138 / 3,228)$ \\
\hline Data are presented as percentage and numbers or means with standard \\
deviation. & \\
\hline
\end{tabular}

Table 2. Lesion characteristics.

\begin{tabular}{|c|c|c|}
\hline \multicolumn{2}{|l|}{ Treated lesions } & 4,383 \\
\hline \multicolumn{2}{|c|}{ Treated lesions per patient } & $1.4 \pm 0.7$ \\
\hline \multicolumn{2}{|c|}{ Diameter stenosis, \% } & $86.0 \pm 11.9$ \\
\hline \multicolumn{2}{|c|}{ Lesion length, mm } & $16.9 \pm 8.9$ \\
\hline \multicolumn{2}{|l|}{ 1-vessel disease } & $41.6 \%(1,345 / 3,231)$ \\
\hline \multicolumn{2}{|l|}{ 2-vessel disease } & $31.0 \%(1,001 / 3,231)$ \\
\hline \multicolumn{2}{|l|}{ 3-vessel disease } & $27.3 \%(881 / 3,231)$ \\
\hline \multirow{4}{*}{$\begin{array}{l}\text { ACC/AHA lesion } \\
\text { classification }\end{array}$} & $A$ & $26.4(1,154 / 4,374)$ \\
\hline & B1 & $37.1(1,623 / 4,374)$ \\
\hline & B2 & $19.6(856 / 4,374)$ \\
\hline & $\mathrm{C}$ & $16.9(741 / 4,374)$ \\
\hline \multirow[t]{11}{*}{ Lesion type } & De novo & $94.3(4,126 / 4,376)$ \\
\hline & Restenosis & $0.5(23 / 4,376)$ \\
\hline & In-stent restenosis & $1.0(42 / 4,376)$ \\
\hline & Bifurcation & $2.9(128 / 4,376)$ \\
\hline & Complete closure & $5.7(248 / 4,376)$ \\
\hline & Acute $(<24 \mathrm{hr})$ & $49.2(122 / 248)$ \\
\hline & Subacute (>24 hr) & $14.9(37 / 248)$ \\
\hline & Chronic & $35.9(89 / 248)$ \\
\hline & Ostial & $0.8(36 / 4,376)$ \\
\hline & Bypass & $0.2(7 / 4,376)$ \\
\hline & Other type & $0.7(31 / 4,376)$ \\
\hline \multicolumn{2}{|l|}{ Severe tortuosity } & $1.2(54 / 4,366)$ \\
\hline \multicolumn{2}{|c|}{ Severe calcification } & $3.6(158 / 4,374)$ \\
\hline \multicolumn{3}{|c|}{$\begin{array}{l}\text { Data are presented as percentage and numbers or means with standard } \\
\text { deviation. ACC: American College of Cardiology; AHA: American Heart } \\
\text { Association }\end{array}$} \\
\hline
\end{tabular}

\section{PROCEDURAL RESULTS}

Pre-treatment was performed in $91.5 \%$ of all lesions, including high-pressure predilatation at $\geq 14$ atm in $43.1 \%$. Overall, $98.2 \%$ of the patients were treated with BVS. In $86.4 \%$ of the treated lesions, BVS were used alone, in $10.7 \%$ metallic stents were used alone, and in $2.9 \%$ BVS and DES were used in a "hybrid approach". The mean diameter of the implanted devices was $3.1 \pm 0.6 \mathrm{~mm}$ and the mean length was $19.7 \pm 6.2 \mathrm{~mm}$. An overlap was performed in $13.8 \%$ of patients. Additional post-dilatation was applied in $71.9 \%$ of the lesions, of which $89.5 \%$ were performed at high pressure ( $\geq 14 \mathrm{~atm})$. Procedural success was achieved in $98.9 \%$.

Of all patients discharged, $97.3 \%$ were on aspirin, $97.6 \%$ on an ADP receptor antagonist (clopidogrel $44.2 \%$, prasugrel $34.0 \%$ and ticagrelor $34.0 \%$ ), and $8.0 \%$ on oral anticoagulation. Further procedural details are provided in Table 3.

\section{SIX-MONTH OUTCOME}

Six-month follow-up was completed in $99.3 \%$ of all patients and the median follow-up duration was 204 (195-230) days. At the sixmonth follow-up, $93.2 \%$ were on aspirin, $93.1 \%$ on an additional

Table 3. Procedural parameters.

\begin{tabular}{|c|c|c|}
\hline \multirow[t]{3}{*}{ Access } & Femoral & $52.1(1,682 / 3,228)$ \\
\hline & Brachial & $0.2(5 / 3,228)$ \\
\hline & Radial & $47.7(1,541 / 3,228)$ \\
\hline \multirow{2}{*}{$\begin{array}{l}\text { Imaging before } \\
\text { intervention }\end{array}$} & Intravascular ultrasound & $3.0(97 / 3,227)$ \\
\hline & $\begin{array}{l}\text { Optical coherence } \\
\text { tomography }\end{array}$ & $4.5(145 / 3,227)$ \\
\hline \multicolumn{2}{|c|}{ Patients with BVS } & $98.2(3,172 / 3,231)$ \\
\hline \multicolumn{2}{|c|}{ Patients with only BVS } & $85.2(2,735 / 3,209)$ \\
\hline \multicolumn{2}{|c|}{ Patients with any metallic stent } & $14.7(474 / 3,231)$ \\
\hline \multicolumn{2}{|c|}{ Patients with BVS and metallic stent } & $13.5(437 / 3,231)$ \\
\hline \multirow{4}{*}{$\begin{array}{l}\text { Number of BVS } \\
\text { per lesion }\end{array}$} & 1 scaffold implanted & $76.0(3,332 / 4,383)$ \\
\hline & 2 scaffolds implanted & $10.1(442 / 4,383)$ \\
\hline & 3 scaffolds implanted & $1.2(53 / 4,383)$ \\
\hline & 4 scaffolds implanted & $0.1(5 / 4,383)$ \\
\hline \multicolumn{2}{|c|}{ Any pre-treatment per lesion } & $91.5(4,005 / 4,378)$ \\
\hline \multicolumn{2}{|c|}{ Predilatation per lesion } & $100.0(4,003 / 4,005)$ \\
\hline \multicolumn{2}{|c|}{ High-pressure balloon } & $43.1(1,718 / 3,990)$ \\
\hline \multicolumn{2}{|c|}{ Maximum balloon diameter, $\mathrm{mm}$} & $2.8 \pm 0.5$ \\
\hline \multicolumn{2}{|c|}{ Maximum balloon pressure, bar } & $13.6 \pm 3.3$ \\
\hline \multicolumn{2}{|c|}{ Cutting balloon } & $3.3(131 / 4,005)$ \\
\hline \multicolumn{2}{|l|}{ Scoring balloon } & $2.9(118 / 4,005)$ \\
\hline \multicolumn{2}{|c|}{ Drug-eluting balloon } & $2.5(102 / 4,005)$ \\
\hline \multicolumn{2}{|c|}{ Rotablation } & $0.1(6 / 4,005)$ \\
\hline \multicolumn{2}{|c|}{ Post-dilatation per lesion } & $71.9(3,146 / 4,375)$ \\
\hline \multicolumn{2}{|c|}{ High-pressure balloon } & $89.5(2,813 / 3,143)$ \\
\hline \multicolumn{2}{|c|}{ Maximum balloon diameter, $\mathrm{mm}$} & $3.3 \pm 0.5$ \\
\hline \multicolumn{2}{|c|}{ Maximum balloon pressure, bar } & $16.7 \pm 4.1$ \\
\hline \multicolumn{2}{|c|}{ Procedure successful } & $98.9(4,330 / 4,377)$ \\
\hline \multicolumn{3}{|c|}{$\begin{array}{l}\text { Data are presented as percentage and numbers or means with standard } \\
\text { deviation. }\end{array}$} \\
\hline
\end{tabular}


ADP receptor antagonist (clopidogrel $44.1 \%$, prasugrel $33.2 \%$, and ticagrelor $22.7 \%$ ), and $8.5 \%$ on oral anticoagulation. The incidences of MACE, TLF and TVF were $4.1 \%, 2.4 \%$ and $3.6 \%$, respectively. The TLR rate was $1.8 \%$, the TVR rate was $3.2 \%$, and the ARCdefined definite scaffold thrombosis rate was $1.0 \%$. Of the 31 definite scaffold thromboses, eight occurred on the same day as the index procedure. An overview of the six-month results is displayed in Table 4 and Kaplan-Meier curves are shown in Figure 1. There were no statistically relevant differences found regarding patients in whom predilatation and post-dilatation was performed in all lesions compared to all other patients. When comparing patients undergoing implantation according to a retrospectively defined implantation protocol (maximum predilatation balloon and maximum BVS diameter ratio of 1:1 and additional post-dilatation with a balloon size of the BVS diameter up to $0.5 \mathrm{~mm}$ above in all lesions), there were also no relevant differences found (Table 5).

\section{COMPARISON OF PATIENTS TREATED BEFORE AND AFTER 1 JANUARY 2015}

A total of 1,759 patients were treated after 1 January 2015; 1,472 had undergone BVS implantation in 2014 or earlier. There were
Table 4. Clinical outcomes after six months.

\begin{tabular}{|c|c|c|}
\hline \multicolumn{2}{|c|}{ Patients with six-month follow-up record } & $99.3(3,207 / 3,231)$ \\
\hline \multicolumn{2}{|c|}{ All-cause mortality } & $1.2(40 / 3,231)$ \\
\hline \multicolumn{2}{|c|}{ Cardiac death } & $0.4(13 / 3,231)$ \\
\hline \multicolumn{2}{|c|}{ Myocardial infarction } & $2.1(67 / 3,231)$ \\
\hline \multicolumn{2}{|c|}{ Target vessel myocardial infarction } & $1.5(49 / 3,231)$ \\
\hline \multirow{3}{*}{$\begin{array}{l}\text { Scaffold } \\
\text { thrombosis }\end{array}$} & Definite & $1.0(31 / 3,172)$ \\
\hline & Probable & $0.5(15 / 3,172)$ \\
\hline & Definite or probable & $1.4(45 / 3,172)$ \\
\hline \multicolumn{2}{|c|}{ Major adverse cardiac events } & $4.1(131 / 3,231)$ \\
\hline \multicolumn{2}{|c|}{ Target lesion failure } & $2.4(78 / 3,231)$ \\
\hline \multicolumn{2}{|c|}{ Target vessel failure } & $3.6(116 / 3,231)$ \\
\hline \multicolumn{2}{|c|}{ Target lesion revascularisation } & $1.8(57 / 3,231)$ \\
\hline \multicolumn{2}{|c|}{ Target vessel revascularisation } & $3.3(104 / 3,231)$ \\
\hline
\end{tabular}

no protocol changes regarding patient selection or implantation technique within the enrolment period. However, a change of the implantation technique can be observed since the proportion of patients with predilatation and post-dilatation in all
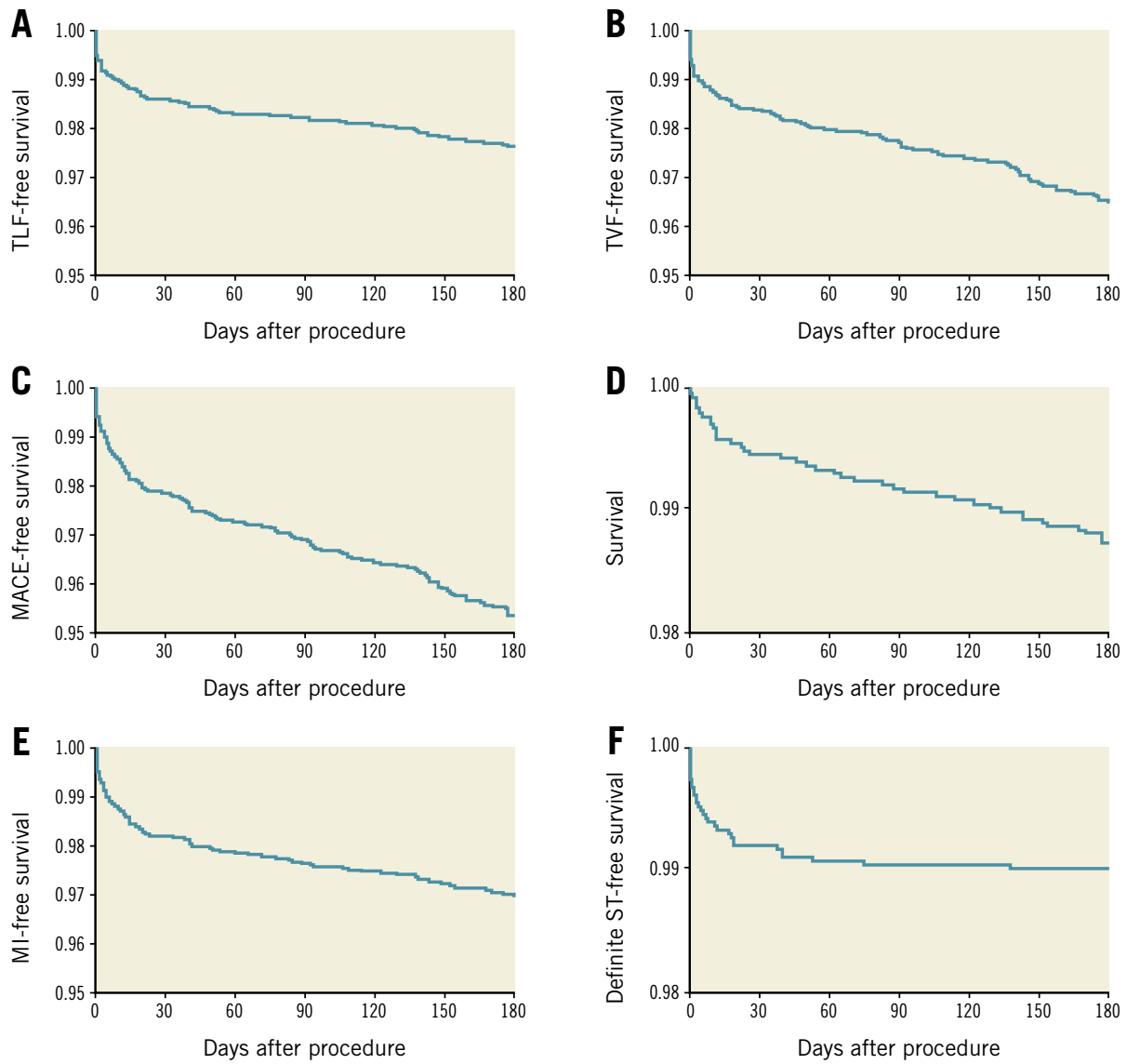

Figure 1. Six-month outcomes of the GABI-R population. A) Kaplan-Meier curves of freedom from target lesion failure (cardiac death, clinically driven target lesion revascularisation or target vessel myocardial infarction). B) Freedom from target vessel failure (cardiac death, target vessel MI, or clinically driven target vessel revascularisation). C) Freedom from major adverse cardiac events (death, target vessel revascularisation, or myocardial infarction). D) Survival. E) Freedom from myocardial infarction. F) Freedom from definite scaffold thrombosis. 
Table 5. Outcomes according to implantation protocol.

\begin{tabular}{|c|c|c|c|c|}
\hline & $\begin{array}{l}\text { Implantation } \\
\text { according to } \\
\text { protocol* }\end{array}$ & Other patients & $p$-value \\
\hline \multicolumn{2}{|c|}{ All-cause mortality } & $1.4(12 / 838)$ & $1.2(27 / 2,334)$ & 0.54 \\
\hline \multicolumn{2}{|c|}{ Cardiac death } & $0.8(7 / 838)$ & $0.3(6 / 2,334)$ & 0.02 \\
\hline \multicolumn{2}{|c|}{ Myocardial infarction } & $2.3(19 / 838)$ & $2.0(47 / 2,334)$ & 0.66 \\
\hline \multicolumn{2}{|c|}{$\begin{array}{l}\text { Target vessel myocardial } \\
\text { infarction }\end{array}$} & $1.8(15 / 838)$ & $1.5(34 / 2,334)$ & 0.50 \\
\hline \multirow{3}{*}{$\begin{array}{l}\text { Scaffold } \\
\text { thrombosis }\end{array}$} & Definite & $1.2(10 / 838)$ & $0.9(21 / 2,334)$ & 0.46 \\
\hline & Probable & $0.5(4 / 838)$ & $0.5(11 / 2,334)$ & 0.98 \\
\hline & $\begin{array}{l}\text { Definite or } \\
\text { probable }\end{array}$ & $1.6(13 / 838)$ & $1.4(32 / 2,334)$ & 0.71 \\
\hline \multicolumn{2}{|c|}{$\begin{array}{l}\text { Major adverse cardiac } \\
\text { events }\end{array}$} & $4.7(39 / 838)$ & $3.9(91 / 2,334)$ & 0.34 \\
\hline \multicolumn{2}{|c|}{ Target lesion failure } & $3.1(26 / 838)$ & $2.2(52 / 2,334)$ & 0.16 \\
\hline \multicolumn{2}{|c|}{ Target vessel failure } & $4.2(35 / 838)$ & $3.5(81 / 2,334)$ & 0.35 \\
\hline \multicolumn{2}{|c|}{$\begin{array}{l}\text { Target lesion } \\
\text { revascularisation }\end{array}$} & $2.2(18 / 838)$ & $1.7(39 / 2,334)$ & 0.37 \\
\hline \multicolumn{2}{|c|}{$\begin{array}{l}\text { Target vessel } \\
\text { revascularisation }\end{array}$} & $3.6(30 / 838)$ & $3.2(74 / 2,334)$ & 0.57 \\
\hline \multicolumn{5}{|c|}{$\begin{array}{l}{ }^{*} \text { The specific implantation protocol was defined as a maximum } \\
\text { predilatation balloon and maximum BVS diameter ratio of } 1: 1 \text { and } \\
\text { additional post-dilatation with a balloon size of the BVS diameter up to } \\
0.5 \mathrm{~mm} \text { above in all lesions. Data are presented as percentage and } \\
\text { numbers. }\end{array}$} \\
\hline
\end{tabular}

treated lesions increased from $58.6 \%$ to $67.4 \%$ ( $p<0.001)$, as well as the proportion of patients who underwent BVS implantation according to a retrospectively defined implantation protocol from $22.2 \%$ to $29.9 \%$ ( $<<0.001)$. When comparing patient characteristics, a slightly less severe cardiovascular risk profile was observed in the group of patients treated in 2015 and later. The clinical presentation was similar in both groups, but patients treated in 2015 and later had less complex lesions. Furthermore, more thorough lesion preparation was performed in 2015 and later. Also, post-dilatation was more often applied with a greater use of high-pressure balloons. Significantly lower six-month event rates occurred during the follow-up of patients treated in 2015 and later (Figure 2). Further details are presented in Table 6.

\section{Discussion}

The present study shows that BVS implantation in daily clinical practice in a heterogeneous patient cohort is associated with high technical success. Midterm outcomes, including six-month followup results, demonstrate clinical safety. Finally, there was a tendency for less complex lesions and a more thorough predilatation and post-dilatation among patients treated in 2015 versus earlier, resulting in significantly lower six-month event rates.

There are currently several randomised controlled trials comparing BVS and DES. In ABSORB III, a total of 2,008 patients were treated with either BVS or an everolimus-eluting DES (XIENCE; Abbott Vascular) ${ }^{11}$. After one year, the TLR rate was $3.0 \%$ and

Table 6. Comparison of patients treated since 2015 and before.

\begin{tabular}{|c|c|c|c|c|}
\hline \multicolumn{2}{|c|}{ Parameter } & $\begin{array}{l}\text { Procedure since } \\
2015\end{array}$ & $\begin{array}{l}\text { Procedure } \\
2013-2014\end{array}$ & $p$-value \\
\hline \multicolumn{5}{|c|}{ Baseline characteristics } \\
\hline \multicolumn{2}{|c|}{ Female gender } & $22.9(402 / 1,759)$ & $23.6(347 / 1,472)$ & 0.63 \\
\hline \multicolumn{2}{|c|}{ Age, years } & $60.9 \pm 11.0$ & $60.5 \pm 11.1$ & 0.02 \\
\hline \multicolumn{2}{|c|}{ Diabetes mellitus } & $20.4(355 / 1,744)$ & $21.8(317 / 1,457)$ & 0.33 \\
\hline \multicolumn{2}{|c|}{ Hyperlipoproteinaemia } & $53.4(899 / 1,683)$ & $60.6(853 / 1,408)$ & $<0.001$ \\
\hline \multicolumn{2}{|c|}{ Hypertension } & $70.9(1,231 / 1,736)$ & $76.1(1,102 / 1,448)$ & $<0.001$ \\
\hline \multicolumn{5}{|c|}{ Clinical presentation } \\
\hline \multicolumn{2}{|c|}{ Acute coronary syndrome } & $52.7(926 / 1,757)$ & $50.1(737 / 1,471)$ & 0.14 \\
\hline \multicolumn{2}{|c|}{ Stable angina } & $32.1(564 / 1,757)$ & $34.7(511 / 1,471)$ & 0.11 \\
\hline \multicolumn{2}{|c|}{ Silent myocardial ischaemia } & $4.6(80 / 1,757)$ & $3.9(58 / 1,471)$ & 0.39 \\
\hline \multicolumn{2}{|c|}{ Other } & $11.3(198 / 1,757)$ & $12.9(190 / 1,471)$ & 0.15 \\
\hline \multicolumn{5}{|c|}{ Lesion characteristics } \\
\hline \multicolumn{2}{|c|}{ De novo vessel } & $95.7(2,274 / 2,376)$ & $92.6(1,852 / 2,000)$ & $<0.001$ \\
\hline \multicolumn{2}{|c|}{ Restenosis } & $0.5(13 / 2,376)$ & $0.5(10 / 2,000)$ & 0.83 \\
\hline \multicolumn{2}{|c|}{ In-stent restenosis } & $0.9(21 / 2,376)$ & $1.1(21 / 2,000)$ & 0.57 \\
\hline \multicolumn{2}{|c|}{ Bifurcation } & $2.2(53 / 2,376)$ & $3.8(75 / 2,000)$ & 0.003 \\
\hline \multicolumn{2}{|c|}{ Complete closure } & $4.8(113 / 2,376)$ & $6.8(135 / 2,000)$ & 0.004 \\
\hline \multicolumn{2}{|c|}{ Acute (<24 hr) } & $48.7(55 / 113)$ & $49.6(67 / 135)$ & 0.88 \\
\hline \multicolumn{2}{|c|}{ Subacute (>24 hr) } & $15.0(17 / 113)$ & $14.8(20 / 135)$ & 0.96 \\
\hline \multicolumn{2}{|c|}{ Chronic } & $36.3(41 / 113)$ & $35.6(48 / 135)$ & 0.91 \\
\hline \multicolumn{2}{|l|}{ 0stial lesion } & $0.5(12 / 2,376)$ & $1.2(24 / 2,000)$ & 0.01 \\
\hline \multicolumn{2}{|l|}{ Bypass } & $0.1(2 / 2,376)$ & $0.3(5 / 2,000)$ & 0.17 \\
\hline $\mathrm{B} 2 / \mathrm{C}$ & & $32.9(781 / 2,376)$ & $40.8(816 / 1,998)$ & $<0.001$ \\
\hline Lesion lengt| & $\mathrm{mm}$ & $17.2 \pm 9.7$ & $17.0 \pm 8.7$ & 0.58 \\
\hline Lesion trea & ment & & & \\
\hline Pre-treated & & $91.0(2,161 / 2,376)$ & $92.1(1,844 / 2,002)$ & 0.17 \\
\hline Predilatation & & $100.0(2,161 / 2,161)$ & $99.9(1,842 / 1,844)$ & 0.13 \\
\hline High-pressur & balloon & $47.7(1,026 / 2,152)$ & $37.6(692 / 1,838)$ & $<0.001$ \\
\hline Cutting ballo & & $4.3(93 / 2,161)$ & $2.1(38 / 1,844)$ & $<0.001$ \\
\hline Scoring ballo & & $4.4(95 / 2,161)$ & $1.2(23 / 1,844)$ & $<0.001$ \\
\hline Drug-eluting & balloon & $3.7(80 / 2,161)$ & $1.2(22 / 1,844)$ & $<0.001$ \\
\hline Rotablation & & $0.0(1 / 2,161)$ & $0.3(5 / 1,844)$ & 0.07 \\
\hline Post-dilatati & & $76.3(1,811 / 2,375)$ & $66.8(1,335 / 2,000)$ & $<0.001$ \\
\hline High-pressur & balloon & $91.7(1,658 / 1,808)$ & $86.5(1,155 / 1,335)$ & $<0.001$ \\
\hline Procedural s & ccess & $98.7(2,344 / 2,376)$ & $99.3(1,986 / 2,001)$ & 0.06 \\
\hline Six-month & utcomes & & & \\
\hline Cardiac deat & & $0.5(9 / 1,759)$ & $0.3(4 / 1,472)$ & 0.28 \\
\hline Myocardial ir & farction & $1.3(23 / 1,759)$ & $3.0(44 / 1,472)$ & $<0.001$ \\
\hline $\begin{array}{l}\text { Target vesse } \\
\text { infarction }\end{array}$ & myocardial & $1.0(18 / 1,759)$ & $2.1(31 / 1,472)$ & 0.01 \\
\hline Scaffold & Definite & $0.6(11 / 1,730)$ & $1.4(20 / 1,442)$ & 0.03 \\
\hline thrombosis & $\begin{array}{l}\text { Definite or } \\
\text { probable }\end{array}$ & $1.0(17 / 1,730)$ & $1.9(28 / 1,442)$ & 0.02 \\
\hline Major advers & cardiac events & $3.1(55 / 1,759)$ & $5.2(76 / 1,472)$ & 0.003 \\
\hline Target lesion & failure & $1.9(33 / 1,759)$ & $3.1(45 / 1,472)$ & 0.03 \\
\hline Target vesse & failure & $3.0(52 / 1,759)$ & $4.4(64 / 1,472)$ & 0.03 \\
\hline Target lesion & evascularisation & $1.2(21 / 1,759)$ & $2.5(36 / 1,472)$ & 0.007 \\
\hline Target vessel & evascularisation & $2.4(42 / 1,759)$ & $4.2(62 / 1,472)$ & 0.003 \\
\hline
\end{tabular}



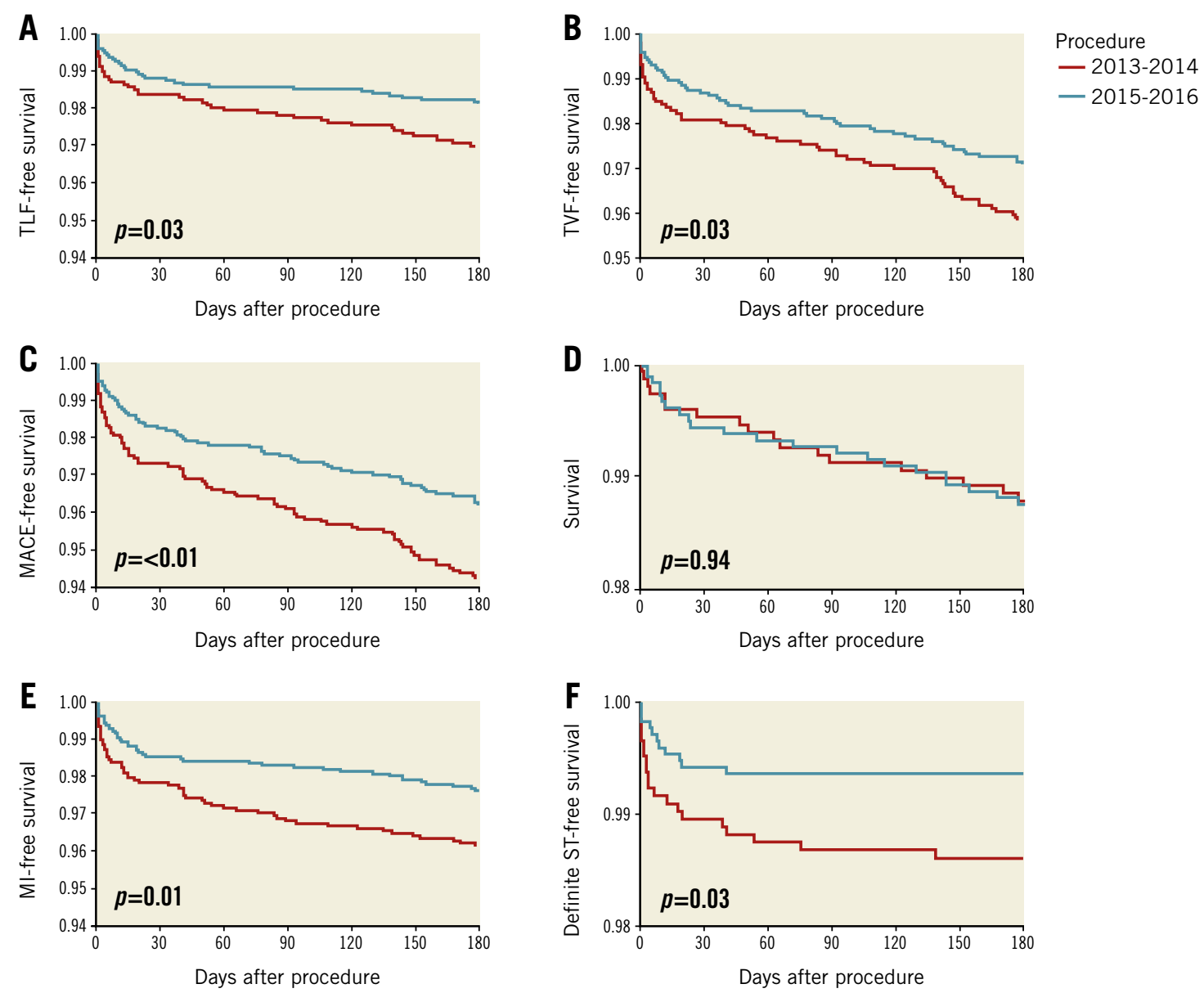

Figure 2. Comparison of outcomes of patients treated in 2015 versus earlier. A) Kaplan-Meier curves comparing freedom from target lesion failure (cardiac death, clinically driven target lesion revascularisation or target vessel myocardial infarction). B) Freedom from target vessel failure (cardiac death, target vessel MI, or clinically driven target vessel revascularisation). C) Freedom from major adverse cardiac events (death, target vessel revascularisation, or myocardial infarction). D) Survival. E) Freedom from myocardial infarction. F) Freedom from definite scaffold thrombosis.

$2.5 \%(\mathrm{p}=0.50)$ in the BVS and XIENCE groups, respectively, whereas scaffold/stent thrombosis occurred in $1.5 \%$ and $0.7 \%$ $(p=0.13)^{12}$. In addition, the AIDA trial evaluated 1,845 patients who were randomised 1:1 to DES. After two years no differences regarding composite endpoints were noted, but a significantly higher definite scaffold thrombosis rate was observed for BVS $(3.1 \% \text { vs. } 0.6 \% ; \mathrm{p}<0.001)^{3}$.

Most of these trials had strict inclusion criteria as patients with acute coronary syndromes were excluded as well as those with lesions $\geq 24 \mathrm{~mm}$ or ostial and bifurcated lesions. Thus, various settings are underrepresented, which reflects the need for all-comers studies without restrictions.

The GHOST-EU registry includes almost 1,200 patients treated with BVS in daily clinical routine. The six-month TLR and scaffold thrombosis rates were $2.5 \%$ and $2.1 \%$, respectively ${ }^{4}$. Notably, the median interval from implantation to scaffold thrombosis was 6.5 days; thus, procedural and non-device-related factors were probably the cause, underscoring the need for optimisation of the implantation technique. The GABI-R RegIstRy demonstrated a lower six-month event rate for TLF (2.4\% vs. $4.4 \%)$ and a lower rate for definite/probable scaffold thrombosis (1.4\% vs. $2.1 \%$ ) than those observed in GHOST-EU ${ }^{4}$. Furthermore, a significant decrease of event rates during 2015 compared to the first two years of GABI-R has been observed. However, the overall scaffold thrombosis rate is still higher than for current DES ${ }^{11,12}$.

Following a learning curve, clinical outcome after BVS implantation may improve ${ }^{6}$, and a refined implantation technique has been shown to reduce event rates ${ }^{13}$. Additionally, an analysis of the GHOST-EU registry demonstrated that systematic predilatation, adequate sizing and systematic post-dilatation ("PSP") are associated with improved outcome ${ }^{5}$. An improvement in the implantation technique has also been observed in GABI-R: for example, the post-dilatation rates per lesion in GHOST-EU, GABI-R 20132014 and GABI-R 2015 were 49\%, 67\% and 76\%, respectively. The analysis of patients treated in 2015 vs. those treated earlier also showed that fewer patients with complex lesions underwent 
BVS implantation. In comparison to GHOST-EU, the proportion of bifurcated lesions, aorto-ostial lesions, and cases of total occlusion and restenosis in GABI-R is substantially lower. It is known that BVS implantation in these complex lesions yields higher event rates $^{14,15}$.

\section{Limitations}

The enrolment was not performed in a randomised fashion. A total of 3,231 patients were included during the protocol-mandated enrolment period, which is less than originally planned. The reasons for that are speculative, but most probably data from several randomised trials published during the enrolment period raised concerns regarding the adverse event rates, especially scaffold thrombosis. In addition, more information on patient and lesion selection was available which further limited the indication for BVS, which is also reflected in the change of lesion and patient characteristics through the course of the enrolment period of this registry. Reimbursement issues in Germany may also have had an influence. Since the discretion to implant a BVS was the only relevant inclusion criterion and left to the physician, BVS use consequently decreased, which led to a premature termination of the registry. Cardiac enzymes were not routinely assessed post PCI in every participating centre and thus under-reporting of periprocedural MI may be possible. Patients who underwent concomitant DES implantation were also included, which may have influenced interpretation of the data. There were no protocol-mandated criteria predefined regarding the selection of either a BVS or a metallic DES and the decision was left to the implanting physician's discretion. One further limitation of this investigation is that there was no specific sizing modality required to support optimal BVS selection. In almost all cases (96.4\%), a visual estimation of the vessel size was performed, which cannot be as precise as online QCA measurements or even intravascular imaging techniques. Routine angiographic followup was not scheduled for patients in this registry, and the analysis does not include any angiographic outcome measurements. The midterm results presented here constitute an interim analysis, and patient follow-up will continue in order to obtain longterm data.

\section{Conclusions}

This analysis of initial results of GABI-R, the largest cohort of unselected patients treated with BVS in clinical routine to date, demonstrates high procedural success and low event rates. However, there is a trend towards a higher rate of scaffold thrombosis as compared with DES registries at six months. A tendency towards treatment of patients with less complex lesions and use of a more dedicated implantation technique during the later phase of the inclusion period was associated with a reduction in event rates. Long-term data regarding safety endpoints as well as quality of life and economic factors will be required to test the proof of concept on different levels; these data will also be provided in time by GABI-R.

\section{Impact on daily practice}

GABI-R is the largest reported patient cohort evaluating BVS implantation in daily routine. Midterm results reveal low event rates, supporting the finding that BVS implantation during daily practice is safe. Within the inclusion period, a trend towards less complex lesions and more dedicated implantation techniques was observed, resulting in lower event rates. This may help to identify optimal patients for BVS implantation.

\section{Acknowledgements}

The authors thank Elizabeth Martinson, $\mathrm{PhD}$, for her editorial assistance, and Dr Steffen Schneider from the Institut für Herzinfarktforschung, Ludwigshafen, Germany, and Dipl.-Ing. Thomas Pfannebecker, Abbott Vascular, for their support and input.

\section{Funding}

The GABI Registry is supported by Abbott Vascular, Santa Clara, CA, USA.

\section{Conflict of interest statement}

H. Nef reports receiving research grants (institutional) and speaker honoraria from Abbott Vascular. G. Richardt is a member of the advisory board of Abbott Vascular. S. Achenbach reports receiving research grants (institutional) from Abbott Vascular and Siemens Healthcare. J. Mehilli has received lecture fees from and is on the advisory board of Abbott Vascular and Terumo, also lecture fees from Lilly/Daiichi Sankyo and Boston Scientific, and an institutional research grant from Abbott Vascular and Edwards Lifesciences. C. Hamm has received speaker honorarium from Medtronic and consulting fees from Abbott Vascular and Medtronic. The other authors have no conflicts of interest to declare.

\section{References}

1. Cassese S, Byrne RA, Ndrepepa G, Kufner S, Wiebe J, Repp J, Schunkert H, Fusaro M, Kimura T, Kastrati A. Everolimus-eluting bioresorbable vascular scaffolds versus everolimus-eluting metallic stents: a meta-analysis of randomised controlled trials. Lancet. 2016;387:537-44.

2. Serruys PW, Chevalier B, Sotomi Y, Cequier A, Carrie D, Piek JJ, Van Boven AJ, Dominici M, Dudek D, McClean D, Helqvist S, Haude M, Reith S, de Sousa Almeida M, Campo G, Iniguez A, Sabaté M, Windecker S, Onuma Y. Comparison of an everolimus-eluting bioresorbable scaffold with an everolimuseluting metallic stent for the treatment of coronary artery stenosis (ABSORB II): a 3 year, randomised, controlled, single-blind, multicentre clinical trial. Lancet. 2016;388:2479-91.

3. Wykrzykowska JJ, Kraak RP, Hofma SH, van der Schaaf RJ, Arkenbout EK, IJsselmuiden AJ, Elias J, van Dongen IM, Tijssen RY, Koch KT, Baan J Jr, Vis MM, de Winter RJ, Piek JJ, Tijssen JG, Henriques JP; AIDA Investigators. Bioresorbable Scaffolds versus Metallic Stents in Routine PCI. N Engl J Med. 2017;376:2319-28. 
4. Capodanno D, Gori T, Nef H, Latib A, Mehilli J, Lesiak M, Caramanno G, Naber C, Di Mario C, Colombo A, Capranzano P, Wiebe J, Araszkiewicz A, Geraci S, Pyxaras S, Mattesini A, Naganuma T, Munzel T, Tamburino C. Percutaneous coronary intervention with everolimus-eluting bioresorbable vascular scaffolds in routine clinical practice: early and midterm outcomes from the European multicentre GHOST-EU registry. EuroIntervention. 2015;10:1144-53.

5. Ortega-Paz L, Capodanno D, Gori $\mathrm{T}$, Nef $\mathrm{H}$, Latib A, Caramanno G, Di Mario C, Naber C, Lesiak M, Capranzano P, Wiebe J, Mehilli J, Araszkiewicz A, Pyxaras S, Mattesini A, Geraci S, Naganuma T, Colombo A, Münzel T, Sabaté M, Tamburino C, Brugaletta S. Predilation, sizing and post-dilation scoring in patients undergoing everolimus-eluting bioresorbable scaffold implantation for prediction of cardiac adverse events: development and internal validation of the PSP score. EuroIntervention. 2017;12:2110-7.

6. Wiebe J, Liebetrau C, Dörr O, Wilkens E, Bauer T, Elsässer A, Achenbach S, Möllmann H, Hamm CW, Nef HM. Impact of the learning curve on procedural results and acute outcome after percutaneous coronary interventions with everolimus-eluting bioresorbable scaffolds in an all-comers population. Cardiovasc Revasc Med. 2015;16:455-60.

7. Nef H, Wiebe J, Achenbach S, Münzel T, Naber C, Richardt G, Mehilli J, Wöhrle J, Neumann T, Biermann J, Zahn R, Kastner J, Schmermund A, Pfannebecker T, Schneider S, Limbourg T, Hamm CW. Evaluation of the short- and long-term safety and therapy outcomes of the everolimus-eluting bioresorbable vascular scaffold system in patients with coronary artery stenosis: Rationale and design of the German-Austrian ABSORB RegIstRy (GABI-R). Cardiovasc Revasc Med. 2016;17:34-7.

8. Tamburino C, Latib A, van Geuns RJ, Sabate M, Mehilli J, Gori T, Achenbach S, Alvarez MP, Nef H, Lesiak M, Di Mario C, Colombo A, Naber CK, Caramanno G, Capranzano P, Brugaletta S, Geraci S, Araszkiewicz A, Mattesini A, Pyxaras SA, Rzeszutko L, Depukat R, Diletti R, Boone E, Capodanno D, Dudek D. Contemporary practice and technical aspects in coronary intervention with bioresorbable scaffolds: a European perspective. EuroIntervention. 2015;11:45-52.

9. Cutlip DE, Windecker S, Mehran R, Boam A, Cohen DJ, van Es GA, Steg PG, Morel MA, Mauri L, Vranckx P, McFadden E,
Lansky A, Hamon M, Krucoff MW, Serruys PW; Academic Research Consortium. Clinical end points in coronary stent trials: a case for standardized definitions. Circulation. 2007;115:2344-51.

10. Puricel S, Arroyo D, Corpataux N, Baeriswyl G, Lehmann S, Kallinikou Z, Muller O, Allard L, Stauffer JC, Togni M, Goy JJ, Cook S. Comparison of everolimus- and biolimus-eluting coronary stents with everolimus-eluting bioresorbable vascular scaffolds. J Am Coll Cardiol. 2015;65:791-801.

11. Serruys PW, Chevalier B, Dudek D, Cequier A, Carrié D, Iniguez A, Dominici M, van der Schaaf RJ, Haude M, Wasungu L, Veldhof S, Peng L, Staehr P, Grundeken MJ, Ishibashi Y, GarciaGarcia HM, Onuma Y. A bioresorbable everolimus-eluting scaffold versus a metallic everolimus-eluting stent for ischaemic heart disease caused by de-novo native coronary artery lesions (ABSORB II): an interim 1-year analysis of clinical and procedural secondary outcomes from a randomised controlled trial. Lancet. 2015;385:43-54.

12. Ellis SG, Kereiakes DJ, Metzger DC, Caputo RP, Rizik DG, Teirstein PS, Litt MR, Kini A, Kabour A, Marx SO, Popma JJ, McGreevy R, Zhang Z, Simonton C, Stone GW; ABSORB III Investigators. Everolimus-Eluting Bioresorbable Scaffolds for Coronary Artery Disease. N Engl J Med. 2015;373:1905-15.

13. Puricel S, Cuculi F, Weissner M, Schmermund A, Jamshidi P, Nyffenegger T, Binder H, Eggebrecht H, Munzel T, Cook S, Gori T. Bioresorbable Coronary Scaffold Thrombosis: Multicenter Comprehensive Analysis of Clinical Presentation, Mechanisms, and Predictors. J Am Coll Cardiol. 2016;67:921-31.

14. Gori T, Wiebe J, Capodanno D, Latib A, Lesiak M, Pyxaras SA, Mehilli J, Caramanno G, Di Mario C, Brugaletta S, Weber J, Capranzano P, Sabate M, Mattesini A, Geraci S, Naber CK, Araszkiewicz A, Colombo A, Tamburino C, Nef H, Münzel T. Early and midterm outcomes of bioresorbable vascular scaffolds for ostial coronary lesions: insights from the GHOST-EU registry. EuroIntervention. 2016;12:e550-6.

15. Kawamoto H, Latib A, Ruparelia N, Miyazaki T, Sticchi A, Naganuma T, Sato K, Figini F, Chieffo A, Carlino M, Montorfano M, Colombo A. Clinical outcomes following bioresorbable scaffold implantation for bifurcation lesions: Overall outcomes and comparison between provisional and planned double stenting strategy. Catheter Cardiovasc Interv. 2015;86:644-52. 